\title{
Prevention and treatment of osteonecrosis of the jaw
}

\author{
Coha Bozena ${ }^{*}$, Holik Hrvoje, Knezevic-Pravecek Marijana
}

Department for Internal Medicine, General Hospital "Dr. Josip Benčević”, Slavonski Brod, Croatia;

*Corresponding Author: bozena.coha@,sb.t-com.hr

Received July 19 ${ }^{\text {th }}, 2011$; revised August $9^{\text {th }}, 2011$; accepted august $24^{\text {th }}, 2011$.

\begin{abstract}
Purpose: to update recommendations for the prevention and treatment of osteonecrosis of the jaw in patients on bisphophonate therapy. Osteonecrosis of the jaw is a rare and serious complication of bisphosphonate therapy. Also, it is one of the important and growing clinical public health issues, because biphosphonates are now more commonly used than before. Bisphophonates are primarly used in the treatment of cancer-related conditions such as bone metastases, hypercalcemia, lytic skeletal lesions. More recently, bisphophonate has been approved for the management of osteoporosis. The etiology and pathogenesis remain unknown, however, two important risk factors have been identified, i.e. the potency and length of bisphosphonate use, and recent dental intervention. Recommendations: prior to the introduction of bisphosphonate therapy, all patients should undergo complete dental examination, any active oral cavity infection should be treated and the potential sites of new infection removed. During bisphosphonate therapy, patients should strictly comply with oral hygiene and avoid any invasive procedure of the oral cavity.
\end{abstract}

Keywords: Bisphosphonates; Osteonecrosis; Jaw

\section{INTRODUCTION}

Osteonecrosis of the jaw is a rare and serious complication of bisphosphonate therapy, first described in the literature in 2003. A patient currently or previously treated with bisphosphonates, having a bone protruding in the maxillofacial region persisting for more than eight weeks, without a history of radiotherapy, can be considered as having osteonecrosis of the jaw associated with bisphosphonates [1]. Bisphophonates are primarly used in the treatment cancer-related conditions such us bone metastases, hypercalcemia, lytic skeletal lesions, but more recently use to treat varied of other skeletal conditions, such as heritable skeletal disorders in children, multiple forms of osteoporosis (juvenile, postmenopausal or involutional, glucocorticoid-induced, transplant-induced, immobility-induced, and androgen-deprivation-related) [2]. According to literature data, the expected risk of jaw osteonecrosis in patients suffering from malignant diseases treated with bisphosphonates ranges from $1 \%$ to $11 \%$, depending on the type, dosage and duration of bisphosphonate therapy, and on dental or oral procedure [2-5]. In patients with multiple myeloma, the prevalence of jaw osteonecrosis has been estimated to $7 \%-10 \%$ [2]. The etiology and pathophysiology of jaw osteonecrosis remain obscure; however, two important risk factors have been identified, i.e. the potency and length of bisphosphonate exposure, and recent dental or oral surgery. Some $70 \%$ of patients developing osteonecrosis of the jaw had a recent dental or oral surgical procedure in their history, whereas spontaneous occurrence of jaw osteonecrosis was recorded in $30 \%$ of patients [4]. The length of bisphosphonate therapy is strongly associated with the occurrence of jaw osteonecrosis. The patients developing jaw osteonecrosis received 35 bisphosphonate infusions on an average, in comparison with 15 infusions in those without jaw osteonecrosis. The prevalence of jaw osteonecrosis was significantly greater after the use of zoledronic acid than after pamidronate $[4,6]$. In their study, Bamias et al. found the expected risk to be $1 \%$ in the first year and $21 \%$ in the third year of treatment with zoledronic acid, whereas for pamidronate it was $0 \%$ in the first two years and $7 \%$ after four years of treatment [6]. The disease more frequently involved the mandible than the maxilla ( $63 \%$ vs $38 \%$ ). Several groups and organizations (American Society of Clinical Oncology (ASCO), Mayo Clinic, and American Association of Oral and Maxillofacial Surgeons (AAOMS)) published guidelines for the prevention and treatment of bisphosphonate-related osteonecrosis of the jaw, and they all agree that prevention is the best approach in the management of this rare and severe complication [1-3]. In 2009, AAOMS published their guidelines for the pre- 
vention and management of bisphosphonate-related osteonecrosis of the jaw [1].

\section{RECOMMENDATIONS FOR PREVENTION}

1) Prior to the introduction of bisphosphonate therapy (if compatible with the patient's condition), the patient should undergo thorough dental examination; extraction of teeth that cannot be saved; elective dentoalveolar surgical procedures should be completed, waiting for bone healing and mucous membrane epithelialization; and the patient should be informed on the importance of strict oral hygiene and possible mucosal trauma, in particular along the lingual region, occurrence of pain, edema or bone protrusion [1-5].

2) Asymptomatic patient on bisphosphonate therapy: strict oral hygiene; avoiding any procedure that may result in bone lesion; a tooth than cannot be cured may be treated by crown removal and endodontic therapy of the remaining roots; avoiding implants; and annual dental examination $[1,3,4]$.

3) Asymptomatic patient on bisphosphonate therapy: elective dentoalveolar surgical procedures may not be contraindicated in this patient category, but they should be informed on the risk of osteonecrosis of the jaw [1,2]. The individuals taking oral bisphosphonates for less than three years and are free from other clinical risk factors need not postpone the scheduled operative procedures in the oral and maxillofacial region[1]. If compatible with the patient condition, in those taking corticosteroids along with bisphosphonates therapy interruption for at least three months prior to the procedure should be considered, and it should not reintroduced until complete wound and bone healing [1]. In patients taking oral bisphosphonates for more than three years, therapy should be discontinued at least three months prior to the procedure and resumed upon complete bone healing [1].

\section{RECOMMENDATIONS FOR TREATMENT}

The aim of treatment in patients diagnosed with osteonecrosis of the jaw is to alleviate pain, control the infection, and reduce progression or development of bone necrosis. Surgical treatment is less efficient than in osteomyelitis or bone osteoradionecrosis, therefore it should be delayed if possible $[1,2]$. Surgical treatment is reserved for patients in the third stage of disease and those with bone sequestration [1]. Elective surgical procedures should be avoided [1].

Specific treatment depends on clinical stage. According to AAOMS, there are four stages of the disease, presented in Table 1. [1]. Discontinuation or reintroduction of bisphosphonate therapy i.v. depends on the assessment of the risk and benefit for the patient, which should be done by a hemato-oncologist and oral/maxillofacial surgeon together [1]. Short-term therapy interruption is of no use, however, if the patient's condition allows it, then long-term therapy discontinuation may prove useful to stabilize osteonecrosis of the jaw, reduce the risk of new sites of bone necrosis and alleviate disease symptoms. In patients with osteonecrosis of the jaw, discontinuation of oral bisphosphonate therapy is associated

Table 1. American Association of Oral and Maxillofacial Surgeons: treatment of bisphosphonate-related osteonecrosis of the jaw according to four stages.

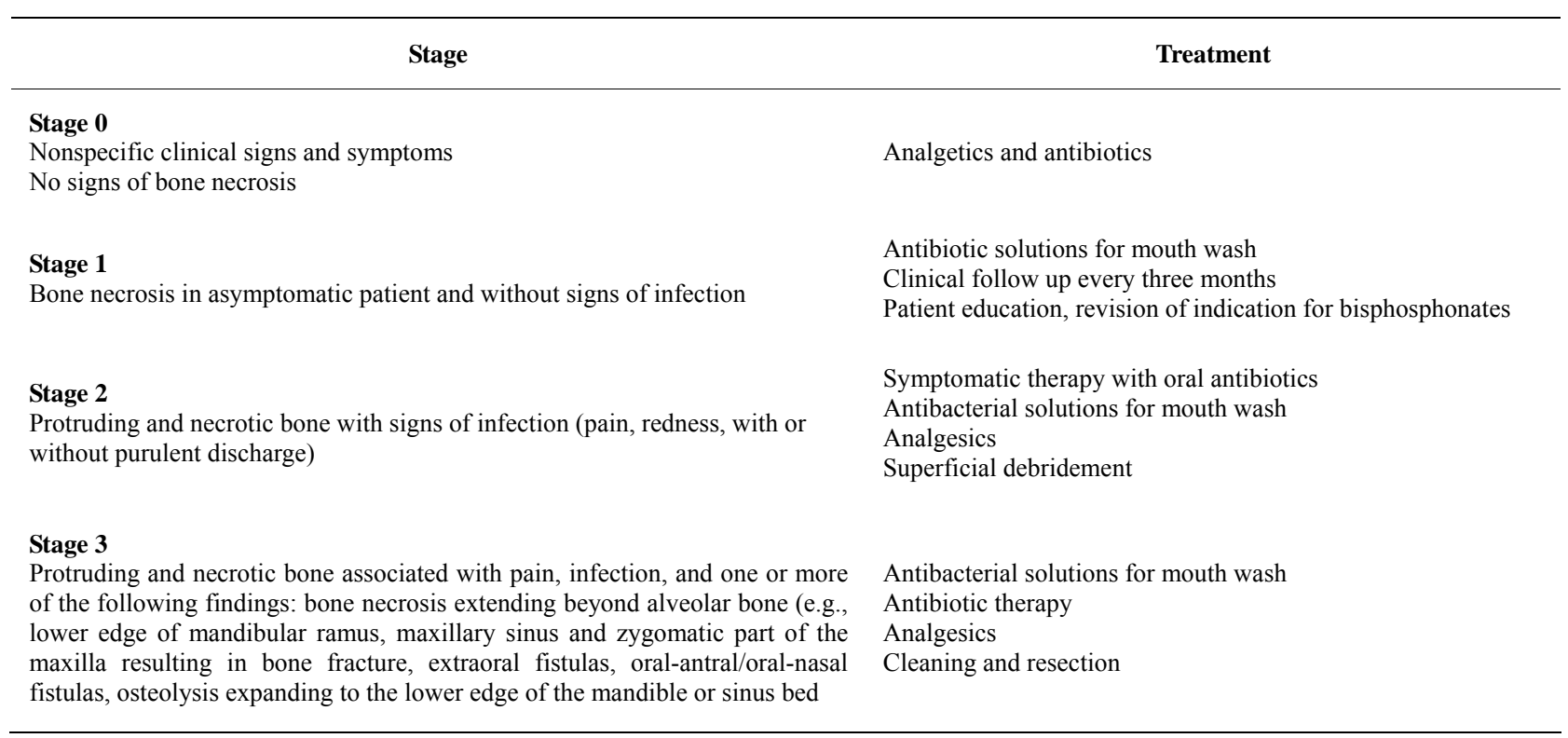


with gradual improvement of clinical status. A 6- to 12-month interruption may lead to spontaneous sequestration or healing following surgical intervention [1].

\section{REFERENCES}

[1] Ruggiero, S.L., Dodson, T.B., Assael, L.A., Landesberg, R., Marx, R.E. and Mehrotra, B. (2009) American Association of Oral and Maxillofacial Surgeons position paper on bisphosphonate-related osteonecrosis of the jaw-2009 update. Australian Endodontic Journal, 35, 119-130.

[2] Drake, M.T., Clarke, B.L. and Khosla, S. (2008) Bisphosphonates: Mechanism of action and role in clinical practice. Mayo Clinic Proceedings, 83, 1032-1045.

[3] Kyle, R.A., Yee, G.C., Somerfield, M.R., Flynn, P.J., Halabi, S., Jagannath, S., Orlowski, R.Z., Roodman, D.G., Twilde, P. and Anderson, K. (2007) American Society of Clinical Oncology 2007 clinical practice guideline update on the role of bisphosphonates in multiple myeloma. Journal of Clinical Oncology, 25, 2464-2472.
[4] Lacy, M.Q., Dispenzieri, A., Gertz, M.A., Greipp, P.R., Gollbach, K.L., Hayman, S.R., Kumar, S., Lust, J.A., Rajkumar, S.V., Russell, S.J., Witzig, T.E., Zeldenrust, S.R., Dingli, D., Bergsagel, P.L., Fonseca, R., Reeder, C.B., Stewart, A.K., Roy, V., Dalton, R.J., Carr, A.B., Kademani, D., Keller, E.E., Viozzi, C.F. and Kyle, R.A. (2006) Mayo clinic consensus statement for the use of bisphosphonates in multiple myeloma. Mayo Clinic Proceedings, 81, 1047-1053.

[5] Terpos, E., Sezer, O., Croucher, P.I., García-Sanz, R., Boccadoro, M., San Miguel, J., Ashcroft, J., Bladé, J., Cavo, M., Delforge, M., Dimopoulos, M.A., Facon, T., Macro, M., Waage, A. and Sonneveld, P. (2009) The use of bisphosphonates in multiple myeloma: Recommendations of an expert panel on behalf of the European Myeloma Network. Annals of Oncology, 20, 1303-1317.

[6] Bamias, A., Kastritis, E., Bamia, C., et al. (2005) Osteonecrosis of the yaw in cancer after treatment with bisphosphonates: incidence and risk factors. Journal of Clinical Oncology, 23, 8580-8587. 